\title{
Hyperspectral Image Denoising Based on Nonlocal Low Rank Dictionary Learning
}

\author{
Zeng ZhiHua*, Zhou Bing and Li Cong \\ City College, Wuhan University of Science and Technology, Hubei Wuhan, 430000, China
}

\begin{abstract}
In allusion to hyperspectral remote sensing image denoising problem, the article proposes an image denoising algorithm based on nonlocal low rand dictionary learning. The basic thought of the algorithm is to make use of the strong correlation among various wavebands of the hyperspectral remote sensing image and meanwhile combine the nonlocal self-similarity and the local sparseness of an image to improve denoising performance. Firstly, combine the strong correlation of waveband images, the nonlocal self-similarity and the local sparseness to establish nonlocal low rank dictionary learning model. Then, adopt iterative method to solve the model to obtain redundant dictionary and sparse representation coefficient. Finally, adopt redundant dictionary and sparse representation coefficient to recover the image. Compared with existing advanced algorithms, due to the adoption of such strong correlation among various wavebands of the hyperspectral image, the algorithm mentioned in the article can well reserve the detailed information of the hyerspectral remote sensing image and improve visual effect. Meanwhile, the test result has verified the effectiveness of the algorithm mentioned in the article.
\end{abstract}

Keywords: Image Denoising, Hyperspectrum, Remote Sensing Image, Low Rank, Sparse Representation.

\section{INTRODUCTION}

Able to accurately and comprehensively describe ground object characteristics, hyperspectral remote sensing image is widely applied in agricultural field, geological prospecting, environmental monitoring, military investigation, etc. However, the severe pollution on hyperspectral remote sensing image during the acquisition and transmission processes can significantly influence the subsequent matching, integration, classification, etc [1]. Therefore, it is importantly significant to research hyperspectral remote sensing image denoising problem $[2,3]$.

Under the assumption that the width of the hyperspectral remote sensing image space is $\mathrm{W}$, the height is $\mathrm{H}$ and the wavebands dimension is $\mathrm{S}$ and the image noise is considered as additive noise [4], so the observation model is:

$\boldsymbol{Y}=\boldsymbol{X}+\boldsymbol{E}$

Therein, $\boldsymbol{Y} \in R^{W \times H \times S}$ is the image polluted by noise, $\boldsymbol{X} \in R^{W \times H \times S}$ is original image, and $\boldsymbol{E} \in R^{W \times H \times S}$ is additive noise [5].

The existing hyperspectral remote sensing image denoising algorithms are basically divided into two types: independent waveband image denoising algorithm and simultaneous waveband image denositing algorithm. Therein, the independent waveband image denoising algorithm includes NLM algorithm, BM3D algorithm, K-SVD algorithm,

\footnotetext{
*Address correspondence to this author at the City College, Wuhan University of Science and Technology, Hubei Wuhan, 430000, China; Tel: 18986139113; E-mail: hunter2011@foxmail.com
}

NCSR algorithm, etc. [6-9], and the basic thought thereof is to regard the hyperspectral images for different wavebands as independent images and then adopt existing denoising algorithm. Specifically, NLM algorithm and BM3D algorithm adopt the nonlocal self-similarity characteristic of the non-adjacent part in the image for denoising, K-SVD algorithm adopts the local sparseness characteristic of the image for denoising, and NCSR adopts both the nonlocal selfsimilarity and the local sparseness for denoising [10-12]. But it is usually difficult for these algorithms to have good performance in practical application, because the internal characteristics of the hyperspectral remote sensing image are neglected in these algorithms. Additionally, the simultaneous waveband image denositing algorithm includes NLM3D algorithm and BM4D algorithm [13, 14], and the basic thought thereof is to adopt the internal characteristics of the multi-band images of the hyperspectral remote sensing image for denoising all waveband images simultaneously. NLM3D algorithm and BM4D algorithm are expanded respectively from NLM algorithm and BM3D algorithm, and aim at adopting the three-dimensional nonlocal selfsimilarity of the hyperspectral remote sensing image for denoising. Compared with independent waveband image denoising algorithms, these algorithms have better effect; but the strong correlation among the waveband images of the the hyperspectral remote sensing image is not considered, so these algorithms still can be further improved.

In consideration of adopting the strong correlation among various waveband images of the hyperspectral remote sensing image and meanwhile combining the nonlocal selfsimilarity and the local sparseness of the image itself, the article proposes an image denoising algorithm based on non- 
local low rank dictionary learning: firstly, establish nonlocal low rank dictionary learning model; then, construct corresponding algorithms to solve the model to realize image denoising. Due to the adoption of such strong correlation among various waveband images, the algorithm mentioned in the article can effectively reserve the texture and the detailed information of various waveband images [15-17].

\section{DICTIONARY LEARNING}

Firstly, simply review the dictionary learning technology. For a given group of samples $\beta=\left(\beta_{1}, \cdots, \beta_{K}\right) \in R^{n \times K}$, the purpose of dictionary learning is to find out the redundant dictionary $\boldsymbol{D} \in R^{n \times m}$ in order to sparsely express each sample as spares representation matrix $\boldsymbol{\Gamma}=\left(\boldsymbol{\Gamma}_{1}, \boldsymbol{\Gamma}_{2}, \mathrm{~L}, \boldsymbol{\Gamma}_{K}\right) \in R^{m \times K}$, and this dictionary learning problem is expressed as:

$\begin{cases}\min _{\boldsymbol{D}, \boldsymbol{x}} & \|\boldsymbol{\beta}-\boldsymbol{D} \boldsymbol{\Gamma}\|_{F}^{2} \\ \text { s.t. } & \left\|\boldsymbol{\Gamma}_{k}\right\|_{0} \leq T_{0} \quad \forall k\end{cases}$

The solving methods for dictionary learning problem (2) usually include MOD algorithm, K-SVD algorithm or online dictionary learning algorithm, etc.

The denoising algorithm in the article is as follows: firstly, establish the nonlocal low rank dictionary learning model for this algorithm; then, construct corresponding algorithms to solve the model to obtain redundant dictionary and sparseness representation coefficient; finally, adopt the results to recover the image $[18,19]$.

\subsection{Nonlocal Low Rank Dictionary Learning Model}

The data structure of the hyperspectral remote sensing image is a cube. It is assumed that the image includes $N$ full-waveband cube data (hereinafter referred to as cube) of which the size is $\sqrt{n} \times \sqrt{n} \times S$ and which are divided by $S$ waveband images from the hyperspectral image, namely $\boldsymbol{p}_{n}(n=1, \cdots, N)$, then the dictionary learning can be expressed as:

$\min _{\boldsymbol{D}, \boldsymbol{\alpha}_{n, s}} \sum_{n=1}^{N} \sum_{s=1}^{S}\left\|\boldsymbol{p}_{n, s}-\boldsymbol{D} \boldsymbol{\alpha}_{n, s}\right\|_{2}^{2}$

s.t. $\left\|\boldsymbol{\alpha}_{n, s}\right\|_{0} \leq T_{0}$

Therein, $\boldsymbol{p}_{n, s} \in R^{n S}$ is the image block vector expression form corresponding to the $S$-th waveband in the $n$-th cube, $\mathrm{D}$ is the redundant dictionary and $\alpha_{n, s}$ is the sparseness representation coefficient [20].

In consideration of adopting nonlocal self-similarity, $\mathrm{K}$ mean value clustering algorithm is used to divide $N$ cube data into $K$ types, then the cube data in each type is used to learn to obtain sub-dictionaries, and then the sub-dictionaries are used to express the data in this type. If the cube data in- cluded in each type is $M_{k}$, then the nonlocal dictionary learning mode of the $k$-th type can be expressed as:

$$
\begin{array}{ll}
\min _{\boldsymbol{D}_{k}, \boldsymbol{\alpha}_{m, s}^{(k)}} & \sum_{m=1}^{M_{k}} \sum_{s=1}^{S}\left\|\boldsymbol{p}_{m, s}^{(k)}-\boldsymbol{D}_{k} \boldsymbol{\alpha}_{m, s}^{(k)}\right\|_{2}^{2} \\
\text { s.t. } & \left\|\boldsymbol{\alpha}_{m, s}^{(k)}\right\|_{0} \leq T_{0}
\end{array}
$$

Therein, $\boldsymbol{p}_{m, s}^{(k)}$ is the image block vector corresponding to the $s$-th waveband of the $m$-th cube in the $k$-th type, $\boldsymbol{D}_{k}$ is the sub-dictionary of the $k$-th type, and $\alpha_{m, s}^{(k)}$ is corresponding sparseness representation coefficient. Since $\boldsymbol{p}_{m, s}^{(k)}$ can be expressed by $\boldsymbol{D}_{k}$, it is indicated that $\left\|\alpha_{m, s}^{(k)}\right\|_{0} \leq T$ can be met. Therefore, the nonlocal dictionary learning model (4) of the $k$-th type is equivalent to:

$$
\min _{\boldsymbol{D}_{k}, \boldsymbol{a}_{m, s}^{(k)}} \sum_{m=1}^{M_{k}} \sum_{s=1}^{S}\left\|\boldsymbol{p}_{m, s}^{(k)}-\boldsymbol{D}_{k} \boldsymbol{\alpha}_{m, s}^{(k)}\right\|_{2}^{2}
$$

Since the waveband images of the hyperspectral image have strong correlation, the coefficient matrix $\alpha_{m}^{(k)}=\left[\alpha_{m, 1}^{(k)}, \cdots, \alpha_{m, s}^{(k)}\right]$ corresponding to the full-waveband data of the $n$-th cube is a low rank matrix. Add the low rank constraint of matrix $\alpha_{m}^{(k)}$ to the nonlocal dictionary learning model () to obtain the nonlocal low rank dictionary learning model as follows [21]:

$$
\min _{\boldsymbol{D}_{k}, \boldsymbol{\alpha}_{m, s}^{(k)}} \sum_{m=1}^{M_{k}} \sum_{s=1}^{S} \frac{\lambda}{2}\left\|\boldsymbol{p}_{m, s}^{(k)}-\boldsymbol{D}_{k} \boldsymbol{\alpha}_{m, s}^{(k)}\right\|_{2}^{2}+\sum_{m=1}^{M_{k}} \operatorname{rank}\left(\boldsymbol{\alpha}_{m}^{(k)}\right)
$$

Therein, $\lambda$ is weighted parameter and $\operatorname{rank}(\bullet)$ is matrix rank. The matrix rank is usually approximated by nuclear norm \|\|$_{*}$ (sum of matrix eigenvalues), so the nonlocal low rank dictionary learning model is equivalent to:

$$
\min _{\boldsymbol{D}_{k}, \alpha_{m, s}^{(k)}} \sum_{m=1}^{M_{k}} \sum_{s=1}^{S} \frac{\lambda}{2}\left\|\boldsymbol{p}_{m, s}^{(k)}-\boldsymbol{D}_{k} \alpha_{m, s}^{(k)}\right\|_{2}^{2}+\sum_{m=1}^{M_{k}}\left\|\alpha_{m}^{(k)}\right\|_{*}
$$

The model is simplified as:

$$
\begin{aligned}
& \min _{\boldsymbol{D}_{k}, \alpha_{m}^{(k)}} \sum_{m=1}^{M_{k}}\left\{\frac{\lambda}{2}\left\|\boldsymbol{p}_{m}^{(k)}-\boldsymbol{D}_{k} \alpha_{m}^{(k)}\right\|_{F}^{2}+\left\|\alpha_{m}^{(k)}\right\|_{*}\right\} \\
& \text { In the matrixes } \quad \boldsymbol{p}_{m}^{(k)}=\left[\boldsymbol{p}_{m, 1}^{(k)}, \cdots, \boldsymbol{p}_{m, S}^{(k)}\right] \\
& \alpha_{m}^{(k)}=\left[\alpha_{m, 1}^{(k)}, \cdots, \alpha_{m, S}^{(k)}\right], \boldsymbol{p}_{n}^{(k)} \text { is the full-waveband data of }
\end{aligned}
$$
the $m$-th cube in the $k$-th type, and $\alpha_{n}^{(k)}$ is the corresponding coefficient matrix. 


\subsection{Solving of Nonlocal Low Rank Dictionary Learning Model}

The nonlocal low rank dictionary learning model is solved basically through two iteration steps: the first step is to fix the sub-dictionary $\boldsymbol{D}_{k}$ and update coefficient matrix $\alpha_{m}^{(k)}$; the second step is to fix coefficient matrix $\alpha_{m}^{(k)}$ and update sub-dictionary $\boldsymbol{D}_{k}$.

Fix the sub-dictionary $\boldsymbol{D}_{k}$ to convert the full-waveband data of each cube $\boldsymbol{p}_{m}^{(k)}\left(m=1,2, \cdots, M_{k}\right)$ into minimization problem

$$
\min _{\boldsymbol{\alpha}_{m}^{(k)}} \frac{\lambda}{2}\left\|\boldsymbol{p}_{m}^{(k)}-\boldsymbol{D}_{k} \boldsymbol{\alpha}_{m}^{(k)}\right\|_{F}^{2}+\left\|\boldsymbol{\alpha}_{m}^{(k)}\right\|_{*}
$$

Then, the optimization problem is actually converted into minimization problem. After auxiliary matrix $\beta$ is introduced, formula (9) is equivalent to

$$
\begin{array}{ll}
\min _{\boldsymbol{\alpha}_{m}^{(k)}} & \frac{\lambda}{2}\left\|\boldsymbol{p}_{m}^{(k)}-\boldsymbol{D}_{k} \boldsymbol{\beta}\right\|_{F}^{2}+\left\|\boldsymbol{\alpha}_{m}^{(k)}\right\|_{*} \\
\text { s.t. } & \boldsymbol{\alpha}_{m}^{(k)}-\boldsymbol{\beta}=0
\end{array}
$$

The corresponding augmented Lagrange function is

$$
\begin{aligned}
& L\left(\boldsymbol{\alpha}_{m}^{(k)}, \boldsymbol{\beta}, \boldsymbol{Z}\right)=\left\|\boldsymbol{\alpha}_{m}^{(k)}\right\|_{*}+\frac{\lambda}{2}\left\|\boldsymbol{p}_{m}^{(k)}-\boldsymbol{D}_{k} \boldsymbol{\beta}\right\|_{F}^{2} \\
& +\left\langle\boldsymbol{Z}, \boldsymbol{\alpha}_{m}^{(k)}-\boldsymbol{\beta}\right\rangle+\frac{\mu}{2}\left\|\boldsymbol{\alpha}_{m}^{(k)}-\boldsymbol{\beta}\right\|_{F}^{2}
\end{aligned}
$$

Therein, $\boldsymbol{Z}$ is Lagrange multiplicator and $\mu>0$ is fixed constant. Then, the minimization problem of formula (11) is converted into the minimization problem of Lagrange function.

$$
\min _{\alpha_{m}^{(k)}, \beta, Z} L\left(\alpha_{m}^{(k)}, \beta, \boldsymbol{Z}\right)
$$

The rapidly converged alternating direction method (ADM) is used to solve the above corresponding optimization problem, with the specific steps as follows. The following iteration format of $\alpha_{m}^{(k)}$ is solved through singular value thresholding (SVT):

$$
\alpha_{m}^{(k)}=\boldsymbol{U} \Gamma_{u^{-1}}(\boldsymbol{S}) \boldsymbol{V}^{T}
$$

Therein, $(U, S, V)=S V D\left(\beta-u^{-1} \boldsymbol{Z}\right), \Gamma_{1 / u}(x)$ is:

$$
\Gamma_{1 / u}(x)= \begin{cases}x-1 / u & \text { if } x>1 / u \\ x+1 / u & \text { if } x<1 / u \\ 0 & \text { other }\end{cases}
$$

Availably, the iteration formats of $\beta$ and $\boldsymbol{Z}$ are

$$
\begin{aligned}
& \boldsymbol{\beta}=\left(u I+\lambda\left(\boldsymbol{D}_{k}\right)^{T} \boldsymbol{D}_{k}\right)^{-1} \\
& \left(u \boldsymbol{\alpha}_{m}^{(k)}-Z+\lambda\left(\boldsymbol{D}_{k}\right)^{T} \boldsymbol{p}_{m}^{(k)}\right) \\
& \boldsymbol{Z}=\boldsymbol{Z}-u\left(\boldsymbol{\alpha}_{m}^{(k)}-\boldsymbol{\beta}\right)
\end{aligned}
$$

The coefficient matrix $\alpha_{m}^{(k)}$ can be obtained through the iteration of formulae (13), (14) and (15).

Through coefficient matrix $\alpha_{m}^{(k)}$, formula (15) is into:

$$
\min _{\boldsymbol{D}_{k}}\left\|\boldsymbol{p}^{(k)}-\boldsymbol{D}_{k} \boldsymbol{\alpha}^{(k)}\right\|_{F}^{2}
$$

Therein, $\boldsymbol{p}^{(k)}=\left[\boldsymbol{p}_{1}^{(k)}, \cdots, \boldsymbol{p}_{M_{k}}^{(k)}\right]$ and $\alpha^{(k)}=\left[\alpha_{1}^{(k)}, \cdots, \alpha_{M_{k}}^{(k)}\right]$. The analytical solution expression of above problem is:

$$
\boldsymbol{D}_{k}=\boldsymbol{p}^{(k)}\left(\boldsymbol{\alpha}^{(k)}\right)^{T}\left(\boldsymbol{\alpha}^{(k)}\left(\boldsymbol{\alpha}^{(k)}\right)^{T}\right)^{-1}
$$

Through the above two steps of iterations, obtain the subdictionary $\boldsymbol{D}_{k}$ of the $k$-th type and the coefficient matrix $\alpha_{m}^{(k)}$ of the full-waveband data $\boldsymbol{p}_{m}^{(k)}$ of the $m$-th cube. Similarly, obtain the sub-dictionaries of all types and the coefficient matrix corresponding to the full-waveband data belonging to the corresponding type of the cube in order to obtain the sub-dictionary $\boldsymbol{D}_{n}$ corresponding to full-waveband data $\boldsymbol{p}_{n}$ of each cube and the corresponding coefficient matrix $\alpha_{n}$.

\subsection{Hyperspectral Remote Sensing Image Denoising}

According to section 2.2, obtain the sub-dictionary $\boldsymbol{D}_{n}$ corresponding to the full-waveband data $\boldsymbol{p}_{n}$ of each cube and the corresponding coefficient matrix $\alpha_{n}$ to estimate the full-waveband data $\hat{\boldsymbol{p}}_{n}=\boldsymbol{D}_{n} \alpha_{n}$ of each recovered cube. Joint the estimated full-waveband data $\hat{\boldsymbol{p}}_{n}$ of the cubes according to the positions thereof and average the overlap parts of the cubes to obtain denoised image $\hat{\boldsymbol{X}}$.

\section{DETAILED STEPS AND ANALYSIS OF ALGO- RITHM}

\subsection{Detailed Steps of Algorithm}

The section will describe the detailed steps of the algorithm in the article, as shown in algorithm 1.

Algorithm 1: denoising algorithm based on nonlocal low rank dictionary learning 
Algorithm input: noisy hyperspectral image $\boldsymbol{Y} \in R^{W \times H \times S}$; Initialization: initial dictionary $\boldsymbol{D}^{(0)}$;

Step 1 Divide hyperspectral image into $N$ mutually overlapped cube full-waveband data $\left\{\boldsymbol{p}_{n}\right\}$;

Step 2 Divide all $\boldsymbol{p}_{n}$ into $K$ types through K-average value clustering algorithm;

Step 3 Solve the nonlocal low rank dictionary learning models (9) of $K$ types to obtain the coefficient matrix $\alpha_{n}$ of $\boldsymbol{p}_{n}$ and the corresponding sub-dictionary $\boldsymbol{D}_{n}$;

Step 4 Estimate the full-waveband data $\hat{\boldsymbol{p}}_{n}=\boldsymbol{D}_{n} \alpha_{n}$ of each cube, joint them according to the positions thereof and average the overlapped parts of the cubes to recover the image $\hat{\boldsymbol{X}}$.

Output result: denoised image $\hat{\boldsymbol{X}}$.

Note of algorithm 1:

(a) Selection of initial dictionary $\boldsymbol{D}^{(0)}$ : such rapidly realized data dictionary as DCT dictionary and wavelet dictionary shall be selected as initial dictionary $\boldsymbol{D}^{(0)}$.

(b) Selection of parameter $\lambda$ : regard the nonlocal low rank dictionary learning problem as multiobjective optimization problem in order to obtain the parameter through $\alpha$-method.

\subsection{Calculation Complexity Analysis}

The calculation quantity of the algorithm in the article is mainly from solving the nonlocal low rank dictionary learning model. The calculation quantity of using AMD algorithm to solve formula (9) is $O\left(\left(n S M_{K}\right)^{3}\right)$ and the calculation quantity of the inversion of formula (16) is also $O\left(\left(n S M_{K}\right)^{3}\right) . \quad N$ times of solving processes are needed, so the total calculation quantity is $O\left(N\left(n S M_{K}\right)^{3}\right)$.

\section{EXPERIMENTAL RESULTS AND ANALYSIS}

The section will verify the algorithm performance through experiments and compare K-SVD algorithm and BM4D algorithm. Experiment 1: compare the visual effects of the actual hyperspectral noise images after denoising; Experiment 2: compare the visual effects of the images artificially added with Gaussian noise after denoising; Experiment 3: present the influence of different noise mean square errors on algorithm performance; Experiment 4: present the influence of number of types on algorithm performance; Experiment 5: present the influence of number of overlapped pixels on algorithm performance.

Hyperspectral remote sensing image Indian Pines and Washington DC Mall are selected for the following experiments, wherein the image of Indian Pines includes 220 wavebands and the image of Washington DC Mall includes 191 wavebands, the cube size is $8 \times 8 \times 7$, the overlapped pixels of adjacent cubes are 7, the number of types is $K=50$, and the number of atoms is 512. All experiments are carried out in the computer with $\mathrm{CPU}$ of dual-core $3.0 \mathrm{GHz}$ and memory of $4 \mathrm{~Gb}$, and the algorithm reestablishment performance is measured by peak signal-to-noise ratio (PSNR).

\section{Experiment 1: Compare with the Visual Effect of Actual Image}

This experiment aims at comparing the denoising visual effects of three algorithms through actual image denoising. Indian Pines data are adopted and the effect of the denoised images of two representative wavebands ---- waveband 1 and waveband 109 is compared in (Fig. 1). According to (Fig. 1), compared with K-SVD algorithm and BM4D algorithm, the image denoised through the algorithm in the article is more detailed and clearer.
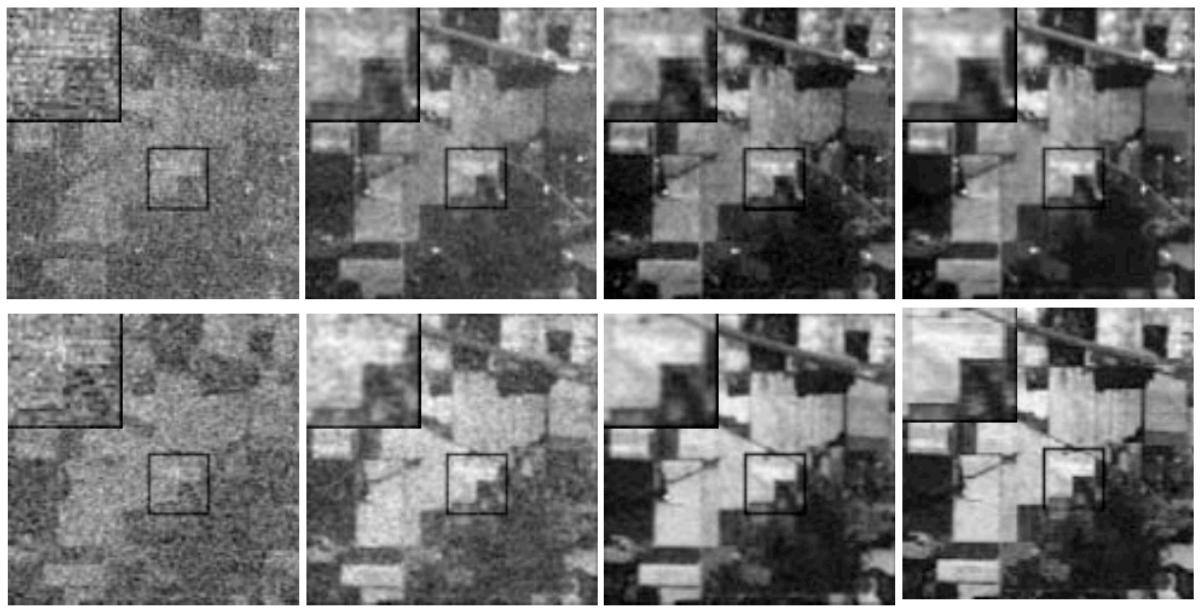

(a) Original Image (b) K-SVD Algorithm (c) BM4D Algorithm (d) Algorithm

Fig. (1). Real hyperspectral remote sensing image to compare the visual effect of noise. 

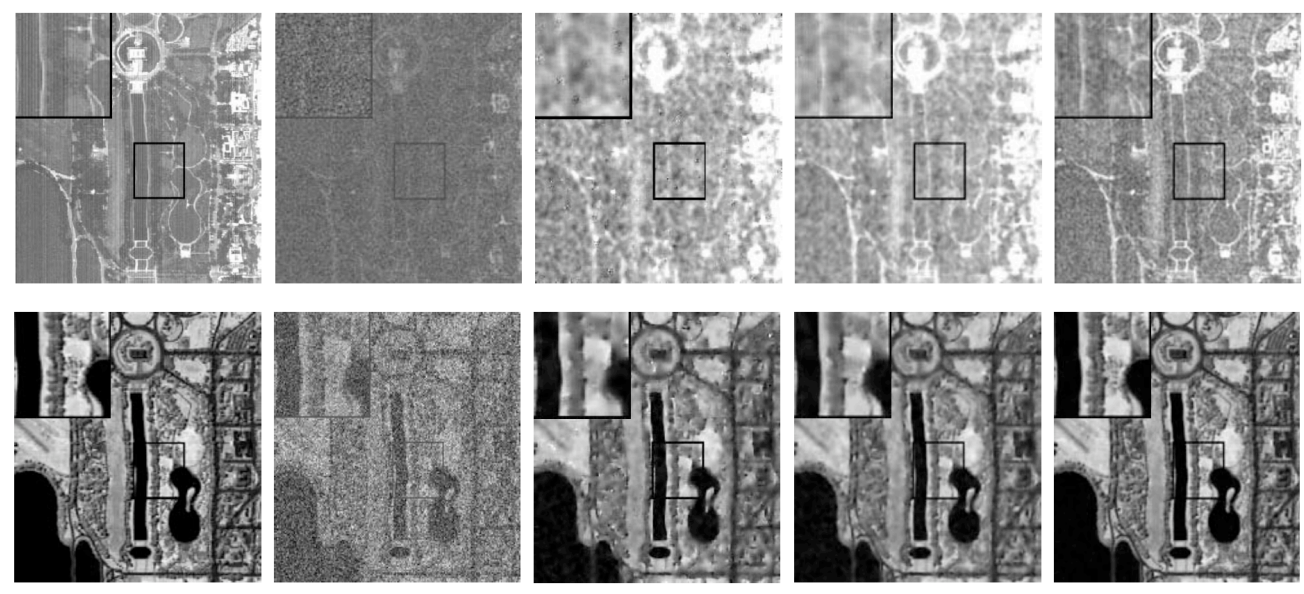

Fig. (2). Comparison of visual effects of gaussian noise eliminated images.

Table 1. Comparison of PSNR values of three algorithms.

\begin{tabular}{|c|c|c|c|c|c|c|}
\hline \multirow{2}{*}{$\sigma$} & \multicolumn{3}{|c|}{ Waveband 1 } & \multicolumn{3}{c|}{ Waveband 100 } \\
\cline { 2 - 7 } & K-SVD Algorithm & BM4D Algorithm & Algorithm in the Article & K-SVD Algorithm & BM4D Algorithm & Algorithm in the Article \\
\hline \hline 20 & 29.19 & 29.81 & 31.13 & 30.34 & 31.03 & 33.26 \\
\hline 30 & 27.28 & 27.31 & 29.50 & 29.03 & 29.21 & 31.49 \\
\hline 50 & 24.33 & 24.82 & 26.78 & 26.59 & 26.29 & 29.76 \\
\hline 100 & 22.17 & 22.79 & 24.86 & 22.82 & 22.75 & 25.58 \\
\hline
\end{tabular}

\section{Experiment 2: Compare with the Visual Effect of the Gaussian Noise Image after Denoising}

This experiment aims at denoising the image with Gaussian noise and accordingly comparing the denoising visual effects of the three algorithms. Washington DC Mall data without noise are added with Gaussian noise, the noise mean square error is taken as $\sigma=30$. The denoising effects of the images of two wavebands (waveband 1 and waveband 100) are compared in (Fig. 1). According to (Fig. 2), compared with other two algorithms, the image denoised through the algorithm in the article is more detailed and clearer, and the visual effect is approximate to the original image.

\section{Experiment 3: Influence of Different Noise Mean Square Errors on Algorithm Performance}

The experiment aims at giving PNSR value of the algorithm under different noise mean square errors. The hyperspectral remote sensing image in Experiment 2 is added with Gaussian noise, and the comparison of PSNR values (for waveband 1 and waveband 100) of K-SVD algorithm, BM4D algorithm and the algorithm in the article under different noise mean square errors are as shown in (Table 1). According to (Table 1), due to the comprehensive consideration of the strong correlation among the images of different wavebands, compared with K-SVD algorithm and BM4D algorithm, the algorithm in the article has significantly improved PSNR value and also has strong noise adaptability.

\section{Experiment 4: Influence of Number of Overlapped Pixels of Cube on Algorithm Performance}

The experiment aims at showing the influence of the number of overlapped pixels of cube full-waveband data (abbreviated as cube) on algorithm performance. The hyperspectral remote sensing image in Experiment 2 is added with Gaussian noise, the mean square errors are respectively as $\sigma=20$ and $\sigma=30$, the number of overlapped pixels of cube is changed from 0 to 7 , and other simulation conditions are not changed. PSNR value (mean value of all waveband images) of the algorithm in the article when the number of the overlapped pixels is changed is as shown in (Fig. 3). According to (Fig. 3), along with the increased number of overlapped pixels, the algorithm performance is gradually improved, and when the number of overlapped pixels is more than or equal to 6 , the algorithm performance is stable.

\section{Experiment 5: Influence of Number of Types $K_{\text {on }}$ Algorithm Performance}

The experiment aims at showing the influence of the number of types on algorithm influence. The hyperspectral remote sensing image in Experiment 2 is added with Gaussian noise, the mean square errors are respectively as $\sigma=20$ and $\sigma=30$, the number of types are from 10 to 100 , and other simulation conditions are not changed. PSNR value (mean value of hyperspectral remote sensing image of all wavebands) of the algorithm in the article when the number of types is changed is as shown in (Fig. 4). According to 
(Fig. 4), when the number of types is between 40 and 80 , the algorithm in the article can obtain relatively good performance, and the small number of types will cause the insufficient similarity of the cubes of the same type while the large number of types will cause insufficient training samples.

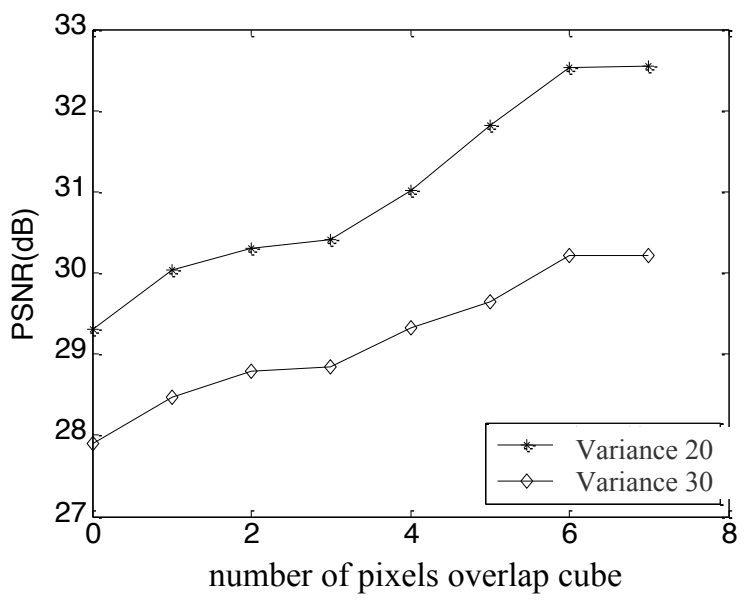

Fig. (3). Influence of overlapped pixels of cube.

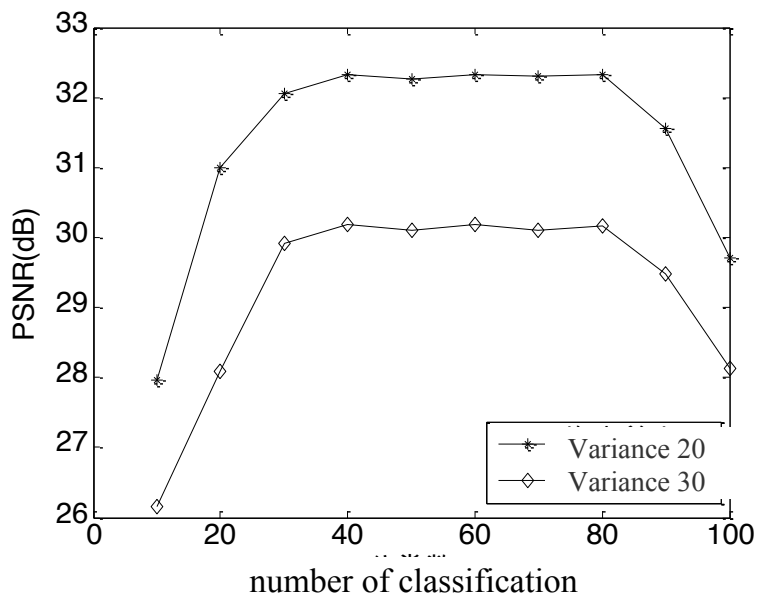

Fig. (4). Influence of number of cube types.

\section{CONCLUSION}

In allusion to hyperspectral remote sensing image denoising problem, the article proposes an image denoising algorithm based on nonlocal low rand dictionary learning. The core thought of the algorithm is to make use of the strong correlation among various waveband images of hyperspectral remote sensing image and meanwhile combine the nonlocal self-similarity and the local sparseness of an image. Due to the adoption of such strong correlation of hyperspectral image, the algorithm mentioned in the article has relatively good effect. The experiment results show that PSNR value of the image recovered by the algorithm in the article is more than that of existing advanced algorithms and can well reserve the detailed information of the image and improve visual effect.

\section{CONFLICT OF INTEREST}

The authors confirm that this article content has no conflicts of interest.

\section{ACKNOWLEDGEMENTS}

Declared none.

\section{REFERENCES}

[1] B. Rasti, J. Sveinsson, and J. Benediktsson, "Hyperspectral image denoising using 3D wavelets, " In: IEEE GRSS, 2012, pp. 13491352.

[2] J. Mairal, F. Bach, J. Ponce, G. Sapiro, and A. Zisserman, “ Nonlocal sparse models for image restoration," IEEE ICCV, 2009, pp. 2272-2279.

[3] J. Dingde, Z. Xu, P. Zhang, and T. Zhu, "A transform domainbased anomaly detection approach to network-wide traffic," Journal of Network and Computer Applications, vol. 15, no. 12, pp. 3736-3745, 2006.

[4] J. He, Y. Geng and K.Pahlavan, "Toward accurate human tracking: modelling time-of-arrival for wireless wearable sensors in multipath environment," IEEE Sensor Journal, vol. 14, no. 11, pp. 39964006, 2014.

[5] J. Wang, L. Zhihan, Z. Xiaolei, F. Jingbao, and C. Ge, "3D graphic engine research based on flash," Henan Science, vol. 4, no. 15, pp. 201-208, 2010

[6] M. Izadi and P. Saeedi, "Robust weighted graph transformation matching for rigid and nonrigid image registration," IEEE Transactions on Image Processing, ,vol. 21, no. 10, pp. 4369-4382, 2012.

[7] M. Maggioni and A. Foi, "Nonlocal transform-domain denoising of volumetric data with group wise adaptive variance estimation," SPIE, vol. 2, no. 3, pp. 45-49, 2012.

[8] M. Maggioni, V. Katkovnik, K. Egiazarian, and A. Foi, "A nonlocal transform domain filter for volumetric data denoising and reconstruction, " IEEE Transactions on Image Processing, vol. 22, no. 1, pp. 119-133, 2013.

[9] M. Zhao, B. An, and Y. Wu, "A robust delaunay triangulation matching for multispectral/ multidate remote sensing image registration," IEEE Geoscience and Remote Sensing Letters, vol. 12, no. 4, pp. 711-715, 2015.

[10] M. Ruina, Z. Lv, Y. Han and Ge Chen, "Research and implementation of geocoding searching and lambert projection transformation based on WebGIS," Geospatial Information, vol. 5, no.13, pp. 102$113,2009$.

[11] S. Tianyun, Z.Lv, S. Gao, X. Li, and H. Lv, “3D seabed: 3D modeling and visualization platform for the seabed," In: IEEE International Conference on Multimedia and Expo Workshops (ICMEW), 2014, pp. 1-6.

[12] T. Alex, B. Laurent, Ma.Piuzzi, Z. Lu, M. Chavent, M. Baaden, and O. Delalande, Advances in Human-Protein InteractionInteractive and Immersive Molecular Simulations, In Tech, 2012.

[13] W. Dong, L. Zhang, and X. Li, "Nonlocally centralized sparse representation for image restoration," In: IEEE Transactions on Image Processing, vol. 22, no. 4, pp. 1620-1630, 2013.

[14] Y. T. Qian, Y. H. Shen, M. C. Ye, and Q. Wang, " 3-D nonlocal means filter with noise estimation for hyperspectral imagery denoising," In: IGARSS, 2012, pp. 356-361.

[15] Y. Geng, and K. Pahlavan, "On the accuracy of RF and image processing based hybrid localization for wireless capsule endoscopy," In: IEEE Wireless Communications and Networking Conference (WCNC), 2015.

[16] Z. Mengxin, Z.Lv, X. Zhang, G. Chen, and K. Zhang, "Research and application of the $3 \mathrm{D}$ virtual community based on WEBVR and RIA," Computer and Information Science, vol. 2, no. 1, p. 84, 2009.

[17] Z. Chen, S. M. Arisona, X. Huang, M. Batty, and G. Schmitt, "Detecting the dynamics of urban structure through spatial network analysis," International Journal of Geographical Information Science, vol. 28, no. 11, pp. 2178-2199, 2014. 
[18] Z. Lv, A. Halawani, S. Feng, S. Rehman, and H. Li, "Touch-less Interactive augmented reality game on vision based wearable device," Personal and Ubiquitous Computing, vol. 2, no. 1, pp. 551$567,2015$.

[19] S. Dang, "Efficient solar power heating system based on lenticular condensation," In: International Conference on Information Science, Electronics and Electrical Engineering (ISEEE), 2014, pp. 2628.
[20] Z. Lv, L. Feng, S. Feng, and H. Li, "Extending touch-less interaction on vision based wearable device," In: IEEE Virtual Reality (VR), 2015.

[21] Y. Geng, J. He, H. Deng and K. Pahlavan, "Modeling the effect of human body on TOA ranging for indoor human tracking with wrist mounted sensor," In: International Symposium on Wireless Personal Multimedia Communications (WPMC), 2013.

Received: June 16, 2015

(C) ZhiHua et al.; Licensee Bentham Open.

This is an open access article licensed under the terms of the Creative Commons Attribution Non-Commercial License (http://creativecommons.org/licenses/by$\mathrm{nc} / 4.0 /$ ) which permits unrestricted, non-commercial use, distribution and reproduction in any medium, provided the work is properly cited. 